\title{
Editorial
}

\section{Synthesis, Properties, and Applications of Single-Domain Magnetic Nanoparticles}

\author{
Subhankar Bedanta, ${ }^{1}$ Anjan Barman, ${ }^{2}$ Wolfgang Kleemann, \\ Oleg Petracic, ${ }^{4}$ and Takeshi Seki ${ }^{5}$ \\ ${ }^{1}$ School of Physical Sciences, National Institute of Science Education and Research (NISER), IOP Campus, \\ Bhubaneswar 751005, India \\ ${ }^{2}$ Department of Condensed Matter Physics and Material Sciences, S. N. Bose National Centre for Basic Sciences, Block JD, \\ Sector III, Salt Lake City, Kolkata 700098, India \\ ${ }^{3}$ Department of Physics, University Duisburg-Essen, 47057 Duisburg, Germany \\ ${ }^{4}$ Juelich Centre for Neutron Science JCNS and Peter Gruenberg Institute (PGI), JARA-FIT, Forschungszentrum Juelich GmbH, \\ 52425 Juelich, Germany \\ ${ }^{5}$ Institute for Materials Research, Tohoku University, 2-1-1 Katahira, Aoba-ku, Sendai 980-8577, Japan
}

Correspondence should be addressed to S. Bedanta; sbedanta@niser.ac.in

Received 11 July 2013; Accepted 11 September 2013

Copyright (C) 2013 S. Bedanta et al. This is an open access article distributed under the Creative Commons Attribution License, which permits unrestricted use, distribution, and reproduction in any medium, provided the original work is properly cited.

Since the pioneering work of Stoner and Wohlfarth over six decades ago, the behavior of magnetic single (or mono) domain particles has held enormous fascination. Magnetization reversal in such single domain particles occurs often via coherent rotation of spins. As a consequence of this rotation mechanism, magnetic nanoparticles show high coercivities, which lie between those of soft and hard permanent magnetic materials. The ability to control the magnetism in these types of particles makes them very attractive for applications, for example, in information storage. Moreover, the research on magnetic nanoparticles has raised hopes for applications in the fields of biology and medicine, for example, drugtargeting, cancer therapy, lymph node imaging, hyperthermia, and so forth.

Detailed information on the properties of single domain nanoparticles is needed before making use of these nanoparticles for any applications. Also the synthesis of such particles by reproducible means is of great importance. The superspins of single-domain particles fluctuate on the timescale modelled within the Néel-Brown theory. The superspins undergo so-called blocking in an ensemble of magnetic nanoparticles with dilute concentration, namely, where inter-particle interactions are negligible. However, in dense ensembles, interaction effects lead to collective states such as superspin glass and superferromagnetism. Several reports on these subjects as well as on superparamagnetism in the very dilute limit have been made in the last decade. This special issue focuses on the preparation, characterization, structural and magnetic properties, and various possible applications of magnetic nanoparticles. It was our hope that it will become an important international platform for researchers to summarize the most recent progress and to exchange ideas in the field of magnetic nanoparticles.

This special issue comprises three review articles. The article by $\mathrm{H}$. Mamiya gives a comprehensive review on progress in understanding the heat dissipation mechanisms of magnetic nanoparticles under large magnetic fields. It also discusses the potential of magnetic nanoparticles in targeted hyperthermia treatment. The review article by $\mathrm{S}$. Mørup et al. outlines realistic models and also experimental studies on spin canting in magnetic nanoparticles. The third review paper written by the editors of this special issue gives a detailed review of various important aspects related to research on magnetic nanoparticles.

The remaining articles in this special issue are research articles, which present selected aspects in reference to synthesis, characterization and also applications of magnetic nanoparticles. The article by Uhm and Rhee explains the synthesis and magnetic properties of $\mathrm{Ni}$ and carbon coated $\mathrm{Ni}$ by a levitational gas condensation method. The article 
by Singh et al. shows the study of the magnetic properties of indirect exchange spring-type $\mathrm{FePt} / \mathrm{M}(\mathrm{Cu}, \mathrm{C}) / \mathrm{Fe}$ trilayered thin films. Krrišt'an et al. have studied the preparation of bentonite/iron oxide composites and characterized them by nuclear magnetic resonance (NMR) and Mössbauer spectroscopy. The article by Quy et al. shows the results on the synthesis of silica-coated $\mathrm{Fe}_{3} \mathrm{O}_{4}$ nanoparticles and their application in the detection of pathogenic viruses. Prodan et al. have studied biological properties of iron oxide nanoparticles, which are obtained in an aqueous suspension. The article by Sato et al. reports on atomic structure imaging of epitaxial $\mathrm{L1}_{0}$-type CoPt nanoparticles using chemically sensitive high-angle annular dark-field scanning transmission electron microscopy. $\mathrm{Fe}_{3} \mathrm{O}_{4}$ nanoparticles and nanowires and their magnetorheological (MR) properties are studied in an article by Rwei et al. The same authors present another study devoted to MR properties of polydimethylsiloxane containing powders of $\mathrm{Fe}_{3} \mathrm{O}_{4}$ and $\mathrm{CoFe}_{2} \mathrm{O}_{4}$ nanoparticles with various shapes and at different compositions and concentrations.

\section{Acknowledgments}

The editors gratefully thank the authors for their contributions to this special issue and the reviewers for their constructive comments and dedication.

Subhankar Bedanta Anjan Barman Wolfgang Kleemann Oleg Petracic Takeshi Seki 

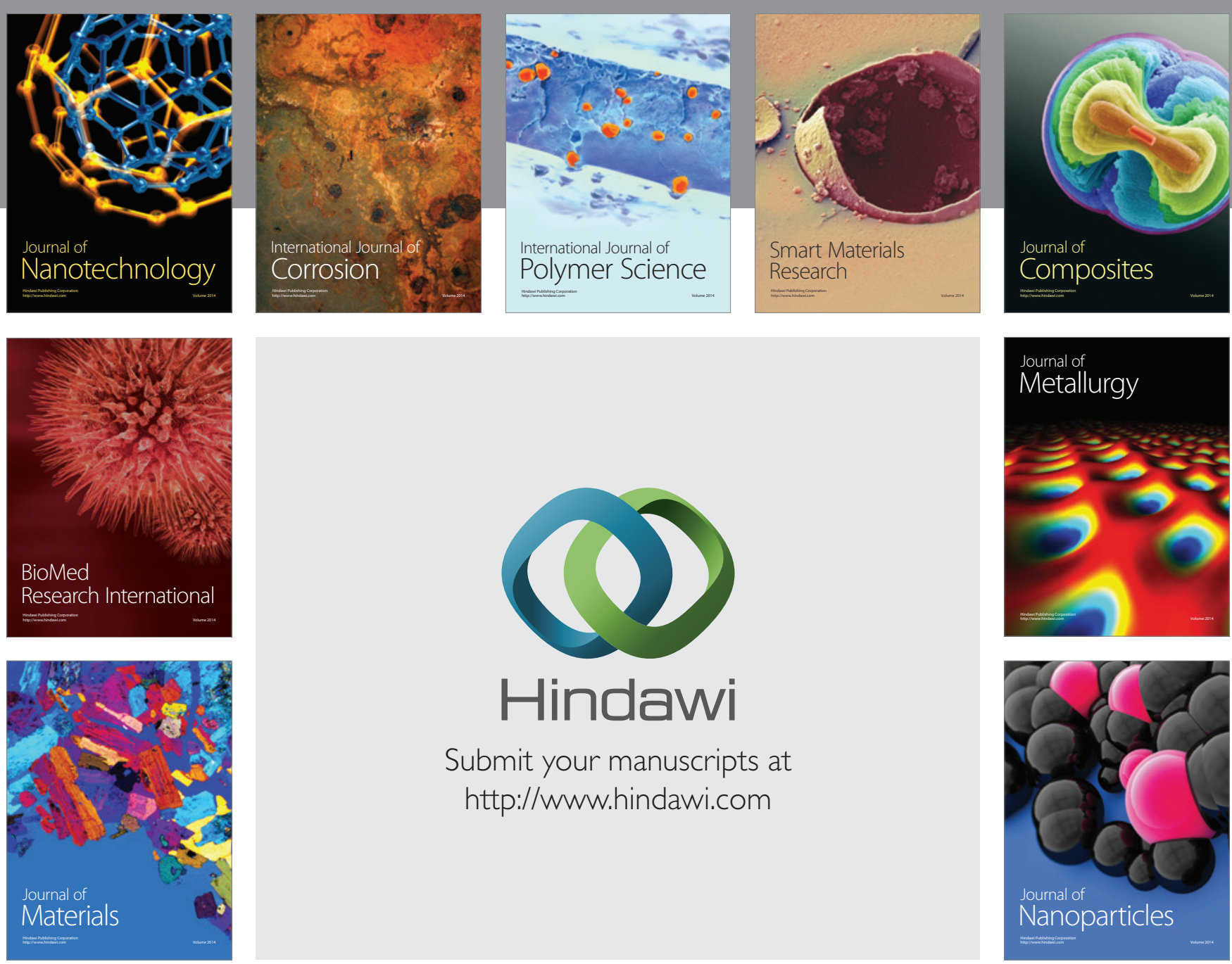

Submit your manuscripts at http://www.hindawi.com
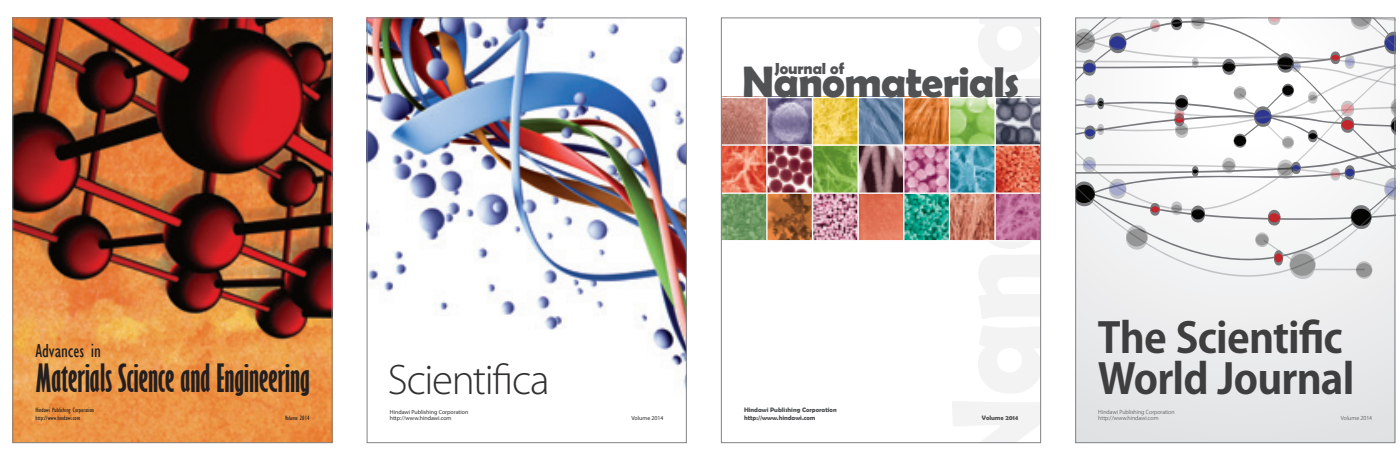

\section{The Scientific World Journal}
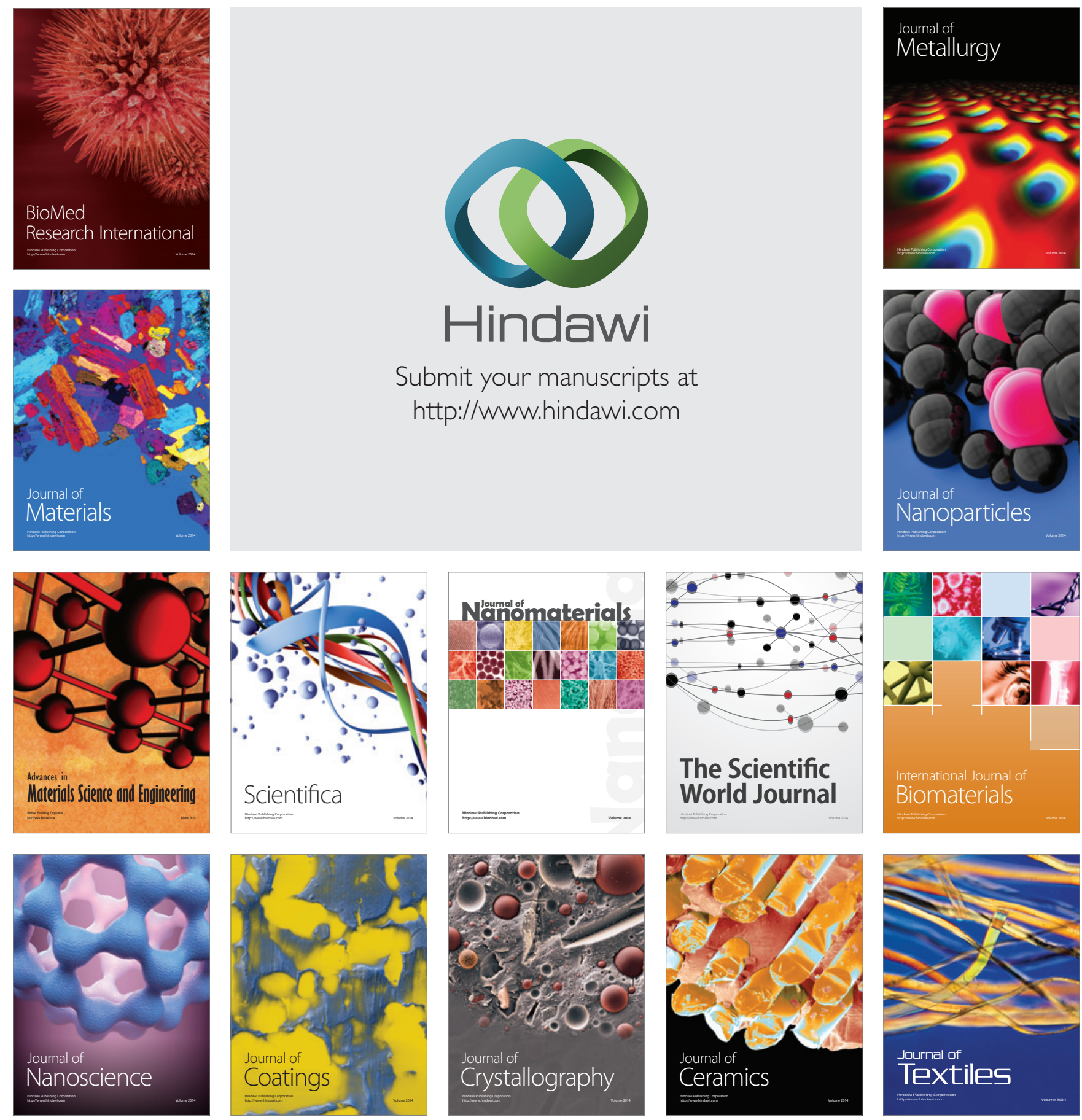